\title{
INYECCIÓN RETROBULBAR DE TRIAMCINOLONA Y CONTROL GLUCÉMICO
}

\section{RETROBULBAR TRIAMCINOLONE INJECTION AND GLYCEMIC CONTROL}

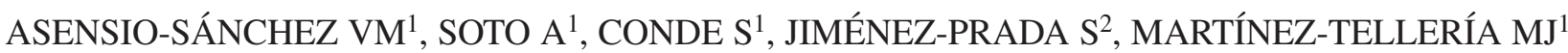

\begin{abstract}
RESUMEN
Objetivo: Estudiar las alteraciones en el control glucémico como efecto secundario sistémico de una inyección retrobulbar de acetónido de triamcinolona en pacientes diabéticos y no diabéticos.

Material y método: Se estudiaron 40 pacientes $(25$ hombres y 15 mujeres, 20 diabéticos y 20 no diabéticos). En cada uno se realizó una inyección retrobulbar con $1 \mathrm{ml}$ de acetónido de triamcinolona (40 mg) y se estudió la glucosa capilar a las 8 de la mañana, en condiciones basales y durante los 7 días post- inyección.

Resultados: La glucosa capilar media fue significativamente mayor en el día 1 post-tratamiento $(218,3$ $\pm 70 \mathrm{mg} / \mathrm{dl})$ que la basal $(138,3 \pm 21 \mathrm{mg} / \mathrm{dl})$ en el grupo diabético $(\mathrm{p}<0,001)$. En el $4^{\circ}$ día post-tratamiento, la glucemia capilar permanecía significativamente elevada comparada con la cifra basal $(185,7 \pm 67,8 \mathrm{mg} / \mathrm{dl})(\mathrm{p}=0,01)$. La glucosa capilar media en el grupo de pacientes no diabéticos fue significativamente mayor en el primer día postinyección $(154,2 \pm 30,1 \mathrm{mg} / \mathrm{dl})$ que la glucemia basal $(119,3 \pm 12 \mathrm{mg} / \mathrm{dl})(\mathrm{p}<0,001) . \mathrm{Al} 4$. $^{\circ}$ día de seguimiento, la glucemia capilar permanecía elevada comparada con la basal en el grupo no diabético $(145,5 \pm 55,8 \mathrm{mg} / \mathrm{dl})(\mathrm{p}=0,01)$.
\end{abstract}

\begin{abstract}
Objective: To look for alterations of glycemic control as a secondary systemic effect of the retrobulbar injection of triamcinolone acetonide in diabetic and non-diabetic patients.

Materials and methods: We studied 40 patients (25 men and 15 women, 20 with and 20 without diabetes). The injection site in all patients was the retrobulbar space, with one injection of $1 \mathrm{ml}$ triamcinolone acetonide $(40 \mathrm{mg})$. Capillary blood glucose was measured at 8 a.m. on the day of treatment as baseline, and on the 7 days following the injection.

Results: The mean capillary blood glucose level was significantly higher at the day 1 post-treatment visit $(218.3 \pm 70 \mathrm{mg} / \mathrm{dl})$ than at baseline $(138.3 \pm 21$ $\mathrm{mg} / \mathrm{dl})$ in the diabetic group $(\mathrm{p}<0.001)$. On day 4 post-treatment, capillary blood glucose remained significantly elevated compared to baseline in the diabetic patients $(185.7 \pm 67.8 \mathrm{mg} / \mathrm{dl})(\mathrm{p}=0.01)$. Mean capillary blood glucose was significantly higher at the day 1 post-treatment visit $(154.2 \pm 30.1$ $\mathrm{mg} / \mathrm{dl})$ than at baseline $(119.3 \pm 12 \mathrm{mg} / \mathrm{dl})$ in the non-diabetic group $(\mathrm{p}<0.001)$. At the day 4 post-treatment visit, capillary blood glucose remained significantly elevated compared to baseline in the nondiabetic patients $(145.5 \pm 55.8 \mathrm{mg} / \mathrm{dl})(\mathrm{p}=0.01)$.
\end{abstract}

\footnotetext{
Recibido: 18/4/08. Aceptado: 14/12/09.

Hospital General Servicio Castellano-Leonés de Salud. Servicio de Oftalmología. Medina del Campo. Valladolid. España.

1 Doctor en Medicina.

2 Licenciado en Medicina.

Correspondencia:

V.M. Asensio Sánchez

España

E-mail: victor_asensio@ orangemail.es
} 
Conclusiones: La administración de una inyección retrobulbar de acetónido de triamcinolona fue seguida por elevaciones en la glucemia capilar en pacientes diabéticos y no diabéticos, pero hiperglucemias severas fueron observadas en el grupo de diabéticos.

Palabras clave: Acetónido de triamcinolona, retrobulbar, glucemia capilar, hiperglucemia, pacientes diabéticos, pacientes no diabéticos.
Conclusions: The administration of triamcinolone acetonide by retrobulbar injection was followed by elevations in capillary glycemia in diabetic and non-diabetic patients, but severe hyperglycemia was observed only in the diabetic group (Arch Soc Esp Oftalmol 2009; 84: 599-604).

Key words: Triamcinolone acetonide, retrobulbar, capillary blood glucose, hyperglycemia, diabetic patients, non-diabetic patients.

\section{INTRODUCCIÓN}

Los corticoides son fármacos muy utilizados en oftalmología para diversas patologías que a veces precisan tratamiento durante un tiempo prolongado $(1,2)$. En estos casos se prefiere la administración local del corticoide para evitar los efectos secundarios de la utilización sistémica continua y a altas dosis $(3,4)$. La administración local de corticoides (peribulbar, retrobulbar) es efectiva en alcanzar altas concentraciones del fármaco en el polo posterior sin aparentes efectos sistémicos secundarios, considerándola tanto oftalmólogos como internistas una forma segura de conseguir concentraciones intraoculares. Los diabéticos, en los que el control glucémico es más lábil y que debe ser más riguroso (5-8), son un gran porcentaje de todos los pacientes de un servicio de oftalmología y presentan un amplio grupo de procesos que afectan al polo posterior y al espacio retro-ocular que son tratados con inyecciones de corticoides muchas veces de forma repetida $(1,2)$. Este estudio pretende analizar las modificaciones de la glucemia en pacientes -diabéticos y no diabéticos- después de una inyección retrobulbar de acetónido de triamcinolona.

\section{SUJETOS, MATERIAL Y MÉTODOS}

Este estudio fue aprobado por el Comité Ético y de Investigación y Ciencia de nuestro hospital. Los pacientes se reclutaron de la consulta de retina y de la policlínica general. Se estudiaron 40 pacientes (20 diabéticos y 20 no diabéticos) entre los años 2006 y 2007. En la tabla I se presentan las características demográficas de ambos grupos. Los criterios de inclusión para los pacientes diabéticos fueron los siguientes:
Tabla I. Características demográficas

\begin{tabular}{lcc}
\hline & Grupo diabético & Grupo No diabético \\
\hline N. & 20 & 20 \\
Sexo & $12(60 \%)$ varones & $13(65 \%)$ varones \\
Edad: & 52,4 D.E. 10,3 & 49,8 D.E. 12,2 \\
\hline \hline
\end{tabular}

DE: desviación estándar.

- Aceptar participar en el estudio con firma del consentimiento informado y poder acudir a todos los controles establecidos.

- No haber sido tratado tres meses previos al estudio con corticoides.

- Diabetes mellitus tipo 1 o tipo 2 tratada por endocrino, internista o médico de atención primaria con glucemias capilares basales medias en ayunas no superiores a $140 \mathrm{mg} / \mathrm{dl}$.

- Permitir manejar todo su historial clínico.

- Pacientes que continúan con el tratamiento ambulatorio habitual de su diabetes.

- Los criterios de inclusión para los pacientes no diabéticos fueron:

- Aceptar participar en el estudio con firma del consentimiento informado y poder acudir a todos los controles establecidos.

- No tener historia pasada o presente de diabetes mellitus.

- No haber sido tratado tres meses previos al estudio con corticoides.

- Glucemias capilares basales no superiores a $120 \mathrm{mg} / \mathrm{dl}$.

- Permitir manejar todo su historial clínico.

\section{Definiciones}

Glucemia capilar basal (o en ayunas): es la que se realiza después de 6 horas sin haber ingerido ningún tipo de alimento. 
Glucemia capilar basal media: consiste en la media de varias determinaciones de glucemia basal. Es muy útil para poder hacer una valoración global que permita realizar cambios más ajustados a la situación.

Paciente clínicamente controlado: paciente con niveles de glucemia basal inferior a $140 \mathrm{mg} / \mathrm{dl}$ en el grupo diabético y a $120 \mathrm{mg} / \mathrm{dl}$ en el grupo no diabético.

Diferencia clínicamente importante: aumento de la glucemia basal superior a $30 \mathrm{mg} / \mathrm{dl}$.

En la tabla II se muestra la indicación de la inyección retrobulbar: se inyectó en la región retrobulbar $1 \mathrm{ml}$ de acetónido de triamcinolona (40 mg, Trigon ${ }^{\circledR}$ depot. Bristol-Myers Squibb, Esplugues, Barcelona). Todas las inyecciones fueron realizadas por la misma persona con una aguja de $25 \mathrm{G}, 40 \mathrm{~mm}$ (Steriseal ${ }^{\circledR}$ ). Después de la inyección retrobulbar no se limitó la actividad diaria de cada paciente y se les instruyó para que realizaran su tratamiento antidiabético correctamente. Se les citó al día siguiente y durante la primera semana después de la inyección (8,00 horas, previo a la inyección de insulina o a la toma de antidiabéticos orales) para realizar una glucemia capilar. Todos los controles capilares fueron realizados con el mismo glucometer (Accu-check Sensor $^{\circledR}$, Roche Diagnostics) con un margen de

Tabla II. Indicación de inyección retrobulbar

\begin{tabular}{|c|c|c|}
\hline & $\begin{array}{c}\text { Grupo diabético } \\
N(\%)\end{array}$ & $\begin{array}{c}\text { Grupo no diabético } \\
\mathrm{N}(\%)\end{array}$ \\
\hline EMCS & $6(30 \%)$ & $0 \quad(0 \%)$ \\
\hline $\mathrm{PsO}$ & $8(40 \%)$ & $5(25 \%)$ \\
\hline DMAE & $5(25 \%)$ & $10(50 \%)$ \\
\hline Traumatismo & $1 \quad(5 \%)$ & $5(25 \%)$ \\
\hline
\end{tabular}

EMCS: Edema macular clínicamente significativo. PsO: Pseudotumor orbitario; DMAE: Degeneración macular asociada a la edad. medición comprendido entre 10-600 mg, y por el mismo personal. La glucemia capilar basal media pre-inyección fue aportada por sus MAP en los pacientes diabéticos y en los no diabéticos fue la media de tres mediciones consecutivas.

El cálculo del tamaño muestral se definió considerando el número de pacientes necesarios para detectar un incremento de $30 \mathrm{mg} / \mathrm{dl}$ en la glucemia a los 7 días tras la inyección retrobulbar. Asumiendo que la desviación típica de la glucemia en pacientes que reciben el tratamiento habitual es de $32,4 \mathrm{mg} / \mathrm{dl}$, con un $80 \%$ de probabilidad de detectar dicha diferencia (si esta existe) y aceptando un error de tipo I (a) $=0,05$, se necesita una muestra de 18 pacientes por grupo de tratamiento. Los resultados obtenidos se han procesado con el programa estadístico SPSS versión 12.02 y se eligió un valor de $\mathrm{p}$ $<0,05$ como nivel de significación estadística. La comparación de dos medias en muestras con datos independientes se ha realizado mediante la aplicación del test no paramétrico de Mann-Whitney. Para la asociación entre dos variables categóricas se usó la prueba de $\chi^{2}$.

\section{RESULTADOS}

Los pacientes fueron divididos en dos grupos en función del antecedente de diabetes. El grupo diabético con 20 pacientes y con una media de edad de 52,4 años (desviación estándar -DE-: 10,3) y el grupo de pacientes no diabéticos formado por 20 personas con una media de edad de 49,8 años (DE: 12,2). La glucemia capilar basal media inicial en el grupo diabético era de $138,3 \pm 21 \mathrm{mg} / \mathrm{dl}$ y en el grupo no diabético de $119,3 \pm 12 \mathrm{mg} / \mathrm{dl}(\mathrm{p}=0,01)$ (tabla III). Se observó un incremento de la glucemia con una diferencia significativa respecto a los valo-

Tabla III. Curva de glucemia capilar en pacientes diabéticos $(\mathbf{n = 2 0})(\mathrm{mg} / \mathrm{dl})$

\begin{tabular}{|c|c|c|c|c|c|c|c|}
\hline Hora & 1. ${ }^{\mathrm{er}} \mathrm{d}$ & $2 .^{\circ} \mathrm{d}$ & $3 .^{\circ} \mathrm{d}$ & $4^{\circ} \mathrm{d}$ & 5. ${ }^{\circ} \mathrm{d}$ & $6^{\circ} \mathrm{d}$ & 7. ${ }^{\circ} \mathrm{d}$ \\
\hline 8 & $218,3 \pm 70$ & $175,5 \pm 41,2$ & $175,3 \pm 42,5$ & $185,7 \pm 67,8$ & $156,2 \pm 53,3$ & $137,5 \pm 38,3$ & $130,4 \pm 29,9$ \\
\hline
\end{tabular}

Glucemia basal: 138,3 \pm 21 . d: día post-inyección. Significación estadística $p<0,001$.

Curva de glucemia capilar en pacientes no diabéticos $(\mathbf{n = 2 0})(\mathbf{m g} / \mathrm{dl})$

\begin{tabular}{|c|c|c|c|c|c|c|c|}
\hline Hora & $1 .^{\mathrm{er}} \mathrm{d}$ & $2 .^{\circ} \mathrm{d}$ & $3 .^{\circ} \mathrm{d}$ & $4^{\circ} \mathrm{d}$ & $5 .^{\circ} \mathrm{d}$ & $6 .^{\circ} \mathrm{d}$ & $7 .^{\circ} \mathrm{d}$ \\
\hline 8 & $154,2 \pm 30,1$ & $155,6 \pm 23,4$ & $145,4 \pm 32,6$ & $145,5 \pm 55,8$ & $134,2 \pm 45,2$ & $131,5 \pm 34,4$ & $123,5 \pm 19,4$ \\
\hline
\end{tabular}

Glucemia basal: 119,3 \pm 12. d: día post-inyección. Significación estadística $\mathrm{p}<0.001$. 
res basales en los dos grupos a las 24 horas de la inyección retrobulbar. La glucosa capilar media fue significativamente mayor en el día 1 post-tratamiento $(218,3 \pm 70 \mathrm{mg} / \mathrm{dl})$ que la basal $(138,3 \pm 21$ $\mathrm{mg} / \mathrm{dl})$ en el grupo diabético $(\mathrm{p}<0,001)$. En el 4. ${ }^{\circ}$ día post-tratamiento, la glucemia capilar permanecía significativamente elevada comparada con la cifra basal $(185,7 \pm 67,8 \mathrm{mg} / \mathrm{dl})(\mathrm{p}=0,01)$. La glucosa capilar media en el grupo de pacientes no diabéticos también fue mayor en el primer día post-inyección $(154,2 \pm 30,1 \mathrm{mg} / \mathrm{dl})$ que la glucemia basal $(119,3 \pm 12 \mathrm{mg} / \mathrm{dl})(\mathrm{p}<0,001)$. Al 4. ${ }^{\circ}$ día de seguimiento, la glucemia capilar permanecía elevada comparada con la basal $(145,5 \pm 55,8 \mathrm{mg} / \mathrm{dl})(\mathrm{p}=0,01)$.

La media de la glucemia basal de los 7 días siguientes a la inyección retrobulbar fue de 168,4 $\mathrm{mg} / \mathrm{dl} \pm 49$ y $141,4 \mathrm{mg} / \mathrm{dl} \pm 34,4$ en el grupo diabético y no diabético respectivamente $(\mathrm{p}=0,01)$. En el grupo no diabético se observó un mejor control de la glucemia durante el seguimiento respecto a los datos basales. En ambos grupos hubo una diferencia estadísticamente significativa con respecto a los niveles basales de glucemia (tabla III). En seis pacientes (30\%) diabéticos las cifras de glucemia superaron los $200 \mathrm{mg} / \mathrm{dl}$, y en uno (5\%) se superaron los $500 \mathrm{mg} / \mathrm{dl}$ necesitando ingreso para su tratamiento. Dentro del grupo diabético, el subgrupo de pacientes con mayor incremento de la glucemia eran los que tenían historia previa de mal control diabético $(\mathrm{p}<0,001)$.

\section{DISCUSIÓN}

La diabetes mellitus es y será uno de los grandes problemas de salud a nivel mundial por el aumento del número de diabéticos y porque es una enfermedad que afecta a múltiples sistemas con consecuencias bioquímicas y anatómicas, por lo que mantener la glucemia dentro del rango de normalidad es el principal objetivo terapéutico (5-8). En este trabajo se ha elegido la glucemia basal, o en ayunas, para el estudio de la alteración de la glucemia porque es la que se realiza después de 6 horas sin haber ingerido ningún tipo de alimento, reflejando las verdaderas alteraciones del metabolismo y la que los pacientes no pueden controlar pues el resto de glucemias las pueden ajustar con insulina rápida. El ojo comprende el $0,01 \%$ del volumen corporal, por lo que la mejor forma de alcanzar concentraciones óptimas de corticoide en el segmento posterior es la aplicación directa o en las cercanías del sitio de acción
$(1,2)$. Los corticoides sistémicos tienen efectos secundarios serios que deben ser controlados cuidadosamente, especialmente en los pacientes diabéticos $(3,4)$. El espacio retrobulbar ha sido utilizado como un reservorio de fármacos para el tratamiento directo de procesos intraoculares pues lo sitúa en la vecindad de la lesión, consiguiendo una biodisponibilidad local mayor que administrándolo de forma sistémica y a dosis comparativamente más bajas, por lo que se supone que los efectos secundarios sistémicos son mínimos. En el tratamiento de procesos inflamatorios del segmento posterior y orbitarios se prefiere el tratamiento local con inyección retrobulbar o subtenoniana para evitar los efectos secundarios sistémicos de los corticoides, con lo que el oftalmólogo está más satisfecho pues aumenta la seguridad del tratamiento pudiendo utilizar dosis más elevadas aplicadas en el foco de la patología, con probables efectos locales pero que el médico oftalmólogo se encuentra más cómodo manejando. El acetónido de triamcinolona es un corticoide de larga acción efectivo cuando se administra intravítreo o retrobulbar $(1,2)$. Su vida media puede ser de hasta 3 meses. Cuando se estudian los efectos adversos de la triamcinolona, además de los efectos secundarios derivados de las técnicas de aplicación, del aumento de la presión intraocular y de la formación de cataratas, no se hace referencia a efectos secundarios sistémicos $(9,10)$. Sin embargo, en el presente trabajo, una inyección retrobulbar de triamcinolona $(40 \mathrm{mg}$ ) produjo cifras muy elevadas de la glucemia capilar, efecto secundario que suele pasar desapercibido para el oftalmólogo y para el internista. En seis pacientes diabéticos (30\%) la glucemia superó los $200 \mathrm{mg} / \mathrm{dl}$ y uno (5\%) precisó hospitalización. En este estudio el análisis global de las glucemias mostró que en ambos grupos los niveles de glucemia se elevaron en más de $30 \mathrm{mg} / \mathrm{dl}$ (margen considerado como clínicamente importante), aunque el grupo de pacientes no diabéticos tuvo un mejor control respecto al grupo de pacientes diabéticos, permaneciendo las diferencias significativas en el valor de la glucemia media a los 4 días después de la inyección retrobulbar. No obstante, en ninguno de los dos grupos se pudo considerar que los pacientes estaban controlados durante los días de seguimiento, con cifras que superaron los $140 \mathrm{mg} / \mathrm{dl}$ en el grupo diabético y los $120 \mathrm{mg} / \mathrm{dl}$ en el grupo no diabético, lo cual no es aceptable, ya que los pacientes se encontraban en un régimen ambulatorio, no polimedicados, sin estrés y hacien- 
do su ejercicio habitual con lo que sí estaban controlados antes de la inyección retrobulbar. La ruta que utiliza la triamcinolona para penetrar en el vítreo puede ser por difusión transescleral o por absorción sistémica del depósito depot retrobulbar. En estudios con animales Bodker et al (11), después de una inyección retrobulbar de dexametasona en un ojo no encontraron diferencias significativas de los niveles intraoculares de dexametasona entre el ojo de la inyección y el adelfo por lo que consideraron que la absorción era esencialmente hematógena. Weijtens $\mathrm{O}$ et al (12), después de una inyección peribulbar de $5 \mathrm{mg}$ de dexametasona fosfato alcanzaron concentraciones séricas de dexametasona similares a las obtenidas con una dosis oral de 7,5 mg considerando que la repetición de este tratamiento puede causar efectos secundarios sistémicos. En pacientes dermatológicos tratados por alopecia areata la terapia más efectiva fue triamcinolona intramuscular, pero también la de más efectos secundarios con supresión adrenocortical en el $23 \%$ frente al $7 \%$ de la terapia corticoidea intravenosa a pulsos (13). Aunque de nuestro estudio no se puede deducir la ruta de penetración intraocular de la triamcinolona retrobulbar, no podemos excluir la ruta sistémica a través de la absorción del depósito depot retroocular, de hecho Weijtens $\mathrm{O}$ et al (14), comprueban que la forma de la curva sérica de la dexametasona después de una inyección peribulbar es semejante a la curva después de un tratamiento intravenoso.

Se puede concluir que la inyección retrobulbar con triamcinolona es una modalidad de corticoterapia sistémica y no debe ser considerada como un simple tratamiento local especialmente en diabéticos y en los pacientes que precisan tratamiento repetido. Pensamos que a los pacientes diabéticos se les debe dar instrucciones para el manejo de su diabetes después de la inyección retrobulbar con corticoides.

\section{BIBLIOGRAFÍA}

1. Gómez-Ulla F, Marticorena J, Alfaro DV 3rd, Fernández M, Méndez ER, Rothen M. Intravitreal triamcinolone for the treatment of diabetic macular edema. Curr Diabetes Rev 2006; 2: 99-112.
2. Jonas JB, Kreissig I, Hugger P, Sauder G, Panda-Jonas S, Degenning $R$. Intravitreal triamcinolone acetonide for exudative age related macular degeneration. $\mathrm{Br} J \mathrm{Oph}$ thalmol 2003; 87: 462-468.

3. Kim HJ, Zhao H, Kitaura H, Bhattacharyya S, Brewe JA, Muglia LP, et al. Glucocorticoids and the osteoclast. Ann N Y Acad Sci 2007; 1116: 335-339.

4. Rosas AL, Kasperlik-Zaluska AA, Papierska L, Bass BL, Pacak K, Eisenhofer G. Pheochromocytoma crisis induced by glucocorticoids: a report of four cases and review of the literature. Eur J Endocrinol 2008; 158: 423-429.

5. Alberti KG, Zimmet PZ. Definition, diagnosis and classification of diabetes mellitus and its complications. Part I: diagnosis and classification of diabetes mellitus provisional report of a WHO consultation. Diabet Med 1998; 15: 539-553.

6. King H, Aubert RE, Herman WH. Global burden of diabetes, 1995-2025: prevalence, numerical estimates and projections. Diabetes Care 1998; 21: 1414-1431.

7. The Diabetes Control and Complications Trial Research Group. The effect of intensive treatment of diabetes on the development and progression of long term complications in insulin-dependent diabetes mellitus. $N$ Engl J Med 1993; 329: 977-986.

8. UK Prospective Diabetes Study (UKPDS) Group. Intensive blood glucose control with sulphonylreas or insulin compared with conventional treatment and risk of complications in patients with type 2 diabetes (UKPDS 33). Lancet 1998; 352: 837-853.

9. Rhee DJ, Peck RE, Belmont J, Martidis A, Liu M, Chang J, et al. Intraocular pressure alterations following intravitreal triamcinolone acetonide. Br J Ophthalmol 2006; 90: 999-1003.

10. Roth DB, Realini T, Feuer WJ, Radhakrishnan R, Gloth J, Heimmel MR, et al. Short-term complications of intravitreal injection of triamcinolone acetonide. Retina 2008; 28: 66-70.

11. Bodker FS, Ticho BH, Feist RM, Lam TT. Intraocular dexamethasone penetration via subconjunctival or retrobulbar injections in rabbits. Ophthalmic Surg 1993; 24: 453-457.

12. Weijtens $O$, van der Sluijs FA, Schoemaker RC, Lentjes EG, Cohen AF, Romijn FP, et al. Peribulbar corticosteroid injection: vitreal and serum concentrations after dexamethasone disodium phosphate injection. Am J Ophthalmol 1997; 123: 358-363.

13. Kurosawa M, Nakagawa S, Mizuashi M, Sasaki Y, Kawamura $M$, Saito $M$, et al. A comparison of the efficacy, relapse rate and side effects among three modalities of systemic corticosteroid therapy for alopecia areata. Dermatology 2006; 212: 361-365.

14. Weijtens $O$, Schoemaker RC, Cohen AF, Romijn FP, Lentjes EG, van Rooij J, et al. Dexamethasone concentration in vitreous and serum after oral administration. Am J Ophthalmol 1998; 125: 673-679. 